\title{
Innovative Approaches to the Treatment of Sleep Apnoea
}

\author{
Silviya Angelova', Julyan Kostadinov', Minko Milev², Gergana Nenova², Mario Milkov³ \\ ${ }^{\prime}$ Medical University of Varna, Bulgaria, Department of Health Economics and Management \\ ${ }^{2}$ Medical College - Medical University of Varna, Bulgaria \\ ${ }^{3}$ Medical University of Varna, Faculty of Dental Medicine
}

\begin{abstract}
Summary:
Innovative approaches to the treatment of a number of important, common and high significance diseases play an increasingly crucial role in modern healthcare and medical services. The use of innovative approaches related to the application of new or improved drug forms, therapies and interventions, or to the introduction of new medical technologies, have improved the quality of healthcare, making treatment more efficient, modern and corresponding to the evolving needs of the patients. Innovative approaches to the treatment of patients with obstructive sleep apnoea syndrome, as the most common type of sleep disorder, which involve the creation of novel medicinal products, technologies and application of new therapies, are a crucial factor for the improvement of patients' health status and for maintaining a high standard of health and patient satisfaction. The use of innovative solutions in the treatment of sleep disorders is an essential condition and a prerequisite for the patients' better quality of life, considering the fact that this is a common disorder, which, according to the World Statistics, affects approximately $4 \%$ of men and $2 \%$ of women aged between 30 and 70 years, and which leads to a higher risk of cardio-vascular diseases and fatality, daytime sleepiness, impaired concentration and alertness, memory disorders and changes in one's personality and behaviour. (1)
\end{abstract}

Key words: innovations, innovative approaches, sleep apnoea, breathing disorder, treatment

\section{Introduction}

Sleep apnoea belongs to a larger group of different breathing disorders during sleep and requires broad differential diagnosis and specialised knowledge in the field of sleep medicine. Apnoea is a major sleep disorder, which occurs when there is a breathing disorder during sleep, which means the presence of a breathing pause with a duration of more than 10 seconds.

There are two types of sleep apnoea: - Obstructive sleep apnoea, which is the most common form of apnoea and which is caused by blocking of the airways, when the soft tissues located at the back of the throat are relaxed during sleep, and - Central sleep apnoea, where, in contrast to obstructive sleep apnoea, the airways are not blocked; it is the brain that "forgets" to transmit a signal for contraction to the respiratory muscles. (2)

\section{Objective}

To present innovative approaches in the treatment of sleep apnoea that result in better health results and improvement in the patients' quality of life.

\section{Materials and methods}

The research on this topic covers content analysis of readings on this issue and an overview of the innovative approaches to the treatment of sleep apnoea. 


\section{Discussion}

There are different treatment methods for sleep apnoea. First, the predisposition factors for obstructive sleep apnoea syndrome should be corrected, such as obesity and nasal septum deviation. In the milder form of this syndrome, medications could be administered before bedtime to reduce the obstructive airway disease, while in the more serious cases, high pressure nocturnal nasal ventilation on spontaneous breathing could be applied by using a nasal mask. Surgical treatment is rarely needed, where in the case of nasal deviation rhinosurgery correction could be applied and, in the case of soft palate obstruction - plastic surgery could be applied. The standard treatment for sleep apnoea includes titration with a CPAP device as the basic non-invasive, diagnostic and therapeutic treatment method, which constitutes a device with a mask used for supply of continuous positive pressure to the airways, titration with a BPAP device - a noninvasive device with a mask used for continuous supply of pressurised air, supporting both the act of inhaling and the act of exhaling, and surgical treatment. (4) Modern sleep medicine increasingly resorts to innovative sleep apnoea treatment methods, such as radiofrequency thermotherapy, laser uvulopalatoplastics, use of robotic pillows to improve sleep, as well as sound-vibration therapy.

1) Radiofrequency thermotherapy - this is the least invasive methodology that allows treatment in outpatient conditions without any risk for the patient. This is a highly efficient innovative technology. The principle of modern radiofrequency surgery is the use of high-frequency /radio/ wave, which results in heating of the tissues and subsequent coagulation. Using a thin electrode, the tissue is precisely punctured at specific sites, which hardens and tightens the enlarged and loose tissue of the soft palate. As a result, the vibration of the soft palate is significantly reduced and the root cause for snoring is eliminated. The success of this method is hidden in the correction of the soft tissue of the palate, which becomes thicker and more elastic as a result of the therapy. The purpose is to eliminate the vibrations of the palate during sleep while keeping its functions at the same time. The modern procedure is comfortable and tolerable for the patient and guarantees over 80 percent improvement. In most cases, 1 to 2 procedures at an interval of 3-4 weeks is sufficient to resolve the problem and repeated intervention is required in only a small share of the cases. The advantages of using radiofrequency thermotherapy are related to the fact that the procedure is performed in outpatient setting and by using local anaesthesia, there are no complications or bleeding, no hospitalisation is necessary and the procedure does not lead to any patient disability - the patient can get back to their normal life dynamics immediately.

2) Laser treatment of snoring (laser uvulopalatoplastics) - where the reason for snoring is the weakness of the soft palate, laser uvulopalatoplastics is a revolutionary solution for handling that problem. The innovative method of laser ablation involves minimum invasion and guaranteed effectiveness. The procedure is not painful for the patient and takes approximately 30 minutes with no need for hospitalisation afterwards. There are no changes in the patient's voice timbre and the patient can immediately get back to their normal routine. The procedure constitutes bloodless surgery involving a one-day hospital stay and is a clear alternative to the classical methods, as it leads to immediate elimination of the symptoms and getting back to normal life.

3) Robotic polar bear pillow - researchers at the "Kabe" laboratory of Waseda University have invented a robot in the form of a white bear, which turns the head of the sleeping person in order to prevent opening of their airways. The bear called Jukusui-Kun (which means "deep sleep") is used as a pillow and has an integrated sensor. When it detects snoring, it raises its paw and gently moves the head of the sleeping person to one side. In this way, the bear can even guarantee a position that stops snoring to people suffering from sleep apnoea. As an addition to the pillow as an innovative and unconventional approach to the treatment of sleep apnoea, a second small bear in the form of a glove monitors the pulse and the blood oxygen level of the sleeping person. When the pillow "hears" snoring and detects a drop in the blood oxygen level of the sleeping person, the paw of the bear comes into action, which first caresses the sleeping person's face with a soft brush attached to the paw and, if this has no effect, it pushes and turns the person's head to one side. There are also special sensors in the linen, which are wireless so that they do not disturb the person's sleep. (7)

4) Sound-vibration therapy - playing the didgeridoo. Didgeridoo is the oldest wind musical instrument used by Australian aborigines more than 
1500 years ago. The observations of a didgeridoo instructor, who reports a decrease in his and his students' daytime sleepiness and snoring after several months of regular practice with the instrument give rise to the idea of studying its treatment effect. The instrument is played by using the technique of continuous air intake. The instrument is played in one tone with a wide range, which can be only compared to human voice. Playing the didgeridoo helps in the treatment of sleep apnoea and mastering the instrument also guarantees the elimination of other breathing problems. According to Puhan et al., the didgeridoo playing technique increases the muscle tone of the upper respiratory tract and reduces their tendency to collapse during sleep. (5) Playing the didgeridoo as a therapy gives positive results with respect to sleepiness assessed according to the Epworth scale, mild sleep disorders and the method is also effective with respect to the apnoea/hypoapnoea index. The data from a randomised controlled trial of Puhan et al. indicates that 4 months of stimulation of the upper respiratory tract by playing the didgeridoo reduces daytime sleepiness in patients suffering from snoring and from a moderately serious obstructive sleep apnoea syndrome. Playing the didgeridoo simulates the innovative treatment methods for obstructive sleep apnoea using neuromuscular simulators. The continuous practice and instrument playing technique that provides exercise to the entire vocal tract and not just to an individual muscle, creates a prerequisite for increasing the tone of the upper respiratory tract. (6)

\section{Conclusion}

In the context of the idea to improve the quality of healthcare, the application of innovative approaches to the treatment of patients with sleep disorders and other conditions is an important element for improving people's overall health profile and quality of life. The application of innovative approaches and solutions to treatment break the stereotypes, because new technologies and practices continuously raise the level in the sphere of healthcare. The use of effective novelties also defines the trends in the development and stimulation of human potential and the development of medicine to achieve better quality of health activities and services as a top priority and an integral component of modern healthcare.

\section{References}

1. American Academy of Sleep Medicine ICSD-2-International classification of sleep disorders Diagnostic and coding manual, 2nd edn. American Academy of Sleep Medicine, 2005

2. Caples SM, Gami AS, Somers VK. Obstructive sleep apnoea. Ann Intern Med. Feb 12005

3. Guilleminault C, Lee JH, Chan A. Pediatric obstructive sleep apnoea syndrome. Arch Pediatr Adolesc Med. Aug 2005

4. Krieger J, Kurtz D, Petiau C, et al. Long-term compliance with CPAP therapy in obstructive sleep apnoea patients and in snorers. Sleep 1996

5. Puhan M, Suarez A, Cascio C, et al. Didgeridoo playing as alternative treatment for obstructive sleep apnoea syndrome: randomised controlled trial. BMJ, doi:10.1136/bmj.38705.470590.55

6. Schwartz A, Smith P, Oliven A Electrical stimulation of the hypoglossal nerve: a potential therapy. J Appl Physiol (1985). 2014 Feb 1; 116(3): $337-344$

7. http://www.techi.com/2011/11/japans-cure-for-snoring-and-sleep-apnea-a-robotic-bear-pillow/ 